\title{
Aproximación a las Curvas de Aprendizaje Individual: Call Center Colombia
}

\author{
Approach to Individual Learning Curves: Call Center Colombia
}

\author{
Francisco Maza-Ávila* \\ Universidad de Cartagena - Colombia \\ ORCID iD: https://orcid.org/0000-0002-3936-8246 \\ fmazaa@unicartagena.edu.co

\section{Ana Karina Romero-Severiche \\ Universidad Pontificia Bolivariana - Colombia \\ ORCID iD: https://orcid.org/0000-0003-2998-0754 \\ anakseveriche@gmail.com}

* Autor a quien debe ser dirigida la correspondencia

\author{
Fecha de recepción: 06/07/2020 \\ Fecha de evaluación: 10/08/2020 \\ Fecha de aceptación: 05/10/2020 \\ Cómo citar: Maza-Ávila, F., \& Romero-Severiche, A. \\ (2020). Aproximación a las Curvas de Aprendizaje Indi- \\ vidual: Call Center Colombia. Revista Cientifica Anfi- \\ bios, 3(2), 22-28. https://doi.org/10.37979/afb.2020v3n2.69
}

\section{Resumen}

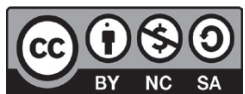

Atribución-NoComercial-CompartirIgual 4.0 Internacional

Este artículo muestra los resultados del cálculo de las curvas de aprendizaje individual para los trabajadores de un centro de llamadas -Call Center- en Colombia, encargado de ofrecer servicios de telefonía móvil. El análisis se realiza con base en la experiencia y el conocimiento adquirido para el desarrollo de su actividad comercial y la relación de éstos con la mejora en la obtención de resultados, según los objetivos de venta planteados. En los resultados empíricos obtenidos se observa que, en la medida que aumenta la experiencia en venta, disminuye el tiempo en las llamadas.

Palabras clave:

Curvas de aprendizaje; centro de llamadas; telefonía móvil; Colombia; organización.

\begin{abstract}
This article shows the results of the calculation of the individual learning curves for the workers of a call center in Colombia, in charge of offering mobile phone services. The analysis is made based on the experience and knowledge acquired for the development of their commercial activity and their relationship with the improvement in obtaining results, according to the sales objectives set. The empirical results obtained show that, as sales experience increases, the time spent on calls decreases.
\end{abstract}

Keywords:

Learning curves; call center; mobile telephony; Colombia; organization. 


\section{Introducción}

Los Centros de Llamadas o Call Center son áreas de una organización $-\mathrm{u}$ organizaciones independientes, que ofrecen sus servicios a otras empresas- que cuentan con personal entrenado para la realización o recepción de llamadas, con la finalidad de ofertar productos o servicios, o para prestar servicios de soporte a clientes individuales o empresariales. Esta figura ha tomado cada día más importancia en los negocios, debido a que suelen ser el canal principal de las empresas para tener un contacto con sus clientes (Aksin, Armony, \& Mehrotra, 2007). Por consiguiente, resulta imprescindible la gestión eficiente las operaciones que se realizan en ellos, con el fin de prestar un mejor servicio en el menor tiempo posible.

Para lograr lo anterior, se considera que el aprendizaje de los agentes o representantes de servicio al cliente de un Call Center es un factor importante, puesto que está asociado con la reducción, a largo plazo, en los tiempos promedio de servicios (Gans, Liu, \& Mandelbaum, 2010). La Curva de Aprendizaje -CA- se constituye en una herramienta importante para la mejora en los tiempos de atención, puesto que permiten evaluar el rendimiento obtenido y planificar con base en sus efectos en la organización. De hecho, el uso de las CA se ha extendido a diferentes sectores industriales, generando nuevos modelos matemáticos para su estimación, según las necesidades específicas de las empresas, logrando corroborar el beneficio de su uso en temas de planeación y control (Lloyd, 1971). De allí el interés de su aplicación en el servicio que ofrecen los Call Center, para así contrastar los efectos del aprendizaje en su operación. Por ello, el principal objetivo de este trabajo consiste en calcular las curvas de aprendizaje individual en los agentes de un centro de llamadas, con el fin de determinar la relación entre el tiempo utilizado para contactar con llamadas con respecto a las ventas realizadas.

Teniendo en cuenta que el análisis se lleva a cabo en un Call Center que inicia la llamada hacia los usuarios con el objetivo de ofertar un servicio de telefonía móvil, es importante medir la eficiencia de las llamadas realizadas por parte de los agentes; la generación de valor de los clientes a quienes se les ofrece el servicio (i.e., si el centro de llamadas produce ganancias monetarias para la empresa); valor generado por cada llamada (i.e., si los agentes del centro de llamadas aprovechan al máximo las oportunidades para vender, actualizar o añadir productos o servicios que los clientes consumen) y si el número de clientes ha aumentado con las ofertas realizadas (Almeida, 2015).

Por lo tanto, para medir el rendimiento operativo de los agentes en este Call Center, se tienen en cuenta lo expuesto anteriormente. Además, se consideran como un buen agente a aquel que tiene un porcentaje de utilización (tiempo de gestión/tiempo de blogueo) igual o superior al $70 \%$, es decir, que ha estado durante ese porcentaje del tiempo marcando; así mismo, debe tener un mayor número de ventas registradas con respecto a las llamadas contactadas (porcentaje de efectividad), lo que se reflejaría en una mayor cantidad de ventas en las horas laboradas.

\section{Revisión de la literatura}

El aprendizaje organizacional, según Levitt \& March (1988), se basa en tres clásicas observaciones extraídas de estudios de comportamiento de las organizaciones. La primera expresa que el comportamiento de una organización se basa en rutinas (Cyert \& March, 1963). La segunda observación señala que las acciones organizacionales son historia-dependientes (Lindblom, 1959), por lo tanto, las rutinas se basan en interpretaciones del pasado más que anticipaciones del futuro. La tercera observación, indica que las organizaciones están orientadas a objetivos (Simon, 1995). Dentro de este marco, las organizaciones ven el aprendizaje como la codificación de inferencias a partir de la historia de las rutinas que guían el comportamiento. En este sentido, el término genérico "rutinas" incluye las formas, reglas, procedimientos, convenciones, estrategias y tecnologías a través de las cuales operan las organizaciones. También, incluye la estructura de creencias, marcos, paradigmas, códigos, las culturas y los conocimientos que refuerzan, elaboran y contradicen las rutinas formales (Levitt \& March, 1988). 
La experiencia organizativa a través de rutinas, permite la experimentación de ensayo y error, dado que la probabilidad de utilizar una rutina aumenta cuando se asocia con éxito en el cumplimiento de un objetivo; o disminuye cuando se asocia con insuficiencia (Cyert \& March, 1963). La organización, por tanto, busca rutinas alternativas para mejorar el desempeño y adopta aquellas rutinas que se consideren mejor que las aplicadas (Levitt \& March, 1988). Lo anterior genera un aprendizaje a través de la experiencia, y la empresa gana experiencia en la medida que se repite una tarea (Argote, 2000; Badiru, 1992; Fioretti, 2007; Schilling, Vidal, Ployhart, \& Marangoni, 2003), así como en la forma en que los miembros de la empresa se familiaricen con la operación y las herramientas (Dar-E1, 2000; Teplitz, 1991). Por consiguiente, el aprendizaje, desde la experiencia, disminuye el tiempo requerido en realizar unidades posteriores a las primeras de las series, o los costos en la ejecución de la operación (Aguirre, 1985; Wright, 1936)

La magnitud de la reducción de tiempo y/o costos, debido a los fenómenos de acomodación o aprendizaje, puede ser predecibles a partir de un modelo matemático que es llamado "curva de aprendizaje" o "curva de experiencia" (Huber, 1991)information distribution, information interpretation, and organizational memory. Este fenómeno de aprender desde la experiencia directa fue analizado por primera vez por Wright (1936) en la industria de aviación norteamericana. En ella observó que los tiempos de trabajo requeridos o empleados en la manufacturación de las primeras unidades de la serie resultan superiores a los tiempos invertidos en la realización de unidades posteriores. Este proceso de "adaptación" daba como resultado que los costos de mano de obra directa disminuían, en la medida que se acumulaba una mayor producción de aeronaves. Al respecto de los hallazgos de Wright, Aguirre (1985) señala que:

"La más significativa conclusión, a nivel operativo, del análisis de los fenómenos de acomodación y aprendizaje es la de los tiempos de ejecución, o lo que es lo mismo, los requerimientos de mano de obra directa por unidad producida en un proceso de fabricación en serie decrecen con un grado de regularidad que es posible predecir".
Múltiples experiencias llevadas a cabo en diversos tipos de industrias permiten afirmar que "(...) cada vez que se duplica el número de unidades producidas de una serie, el tiempo medio de trabajo empleado por unidad de producto disminuye prácticamente en un porcentaje constante" (Nadier \& Smith, 1964). De allí que Wright (1936) exponga un modelo matemático de decrecimiento "lineal" de los tiempos medios. En dicho modelo plantea que si un trabajador realiza la misma tarea varias veces, el tiempo requerido para finalizar esa tarea disminuye a una velocidad constante, expresado por la función:

$$
y=a \cdot x^{\wedge} b
$$

Donde:

y : tiempo (costo) medio requerido para manufaacturar cualquiera de las x unidaades

de una serie de producción

a:tiempo (costo) empleado en la fabricación de la primera unidad de la serie

x:es número de unidades fabricadas

b:es la pendiente de la curva de aprendizaje o también la elasticidad del mejoramiento de la ejecución

Así mismo, la expresión de la ecuación (1) en términos de logaritmos será:

$$
\log \mathrm{y}=\log \mathrm{a}-\mathrm{b} \log \mathrm{x}(2)
$$

Siguiendo el planteamiento expuesto por Wright (1936), se considera importante el estudio de Curvas de Aprendizaje Individual -CAI, aplicados en la producción, vistas como un tipo de transferencia de conocimiento, puesto que la curva de aprendizaje en una persona puede definir el rendimiento de ésta, derivado del nuevo aprendizaje adquirido y de la transferencia del aprendizaje del trabajador anterior a él (Ellis, 1965). Asimismo, se debe tener en cuenta que las CAI pueden hacer referencia al proceso de adquisición de habilidades por parte de cada trabajador, resultado de la experiencia vivida (Glover, 1966). 
Teniendo en cuenta que la rutina es significativa en la construcción de aprendizaje, con respecto a las CAI, puede observarse un aumento del aprendizaje de individuo en la tarea que realiza repetitivamente (Towill \& Bevis, 1972). De igual modo, la formulación estándar de las curvas de aprendizaje es modelada como una función de la experiencia acumulativa en una tarea en particular, en lugar de una serie de tareas, ya que se parte del supuesto que la tasa de aprendizaje se maximiza mediante la dedicación a una sola actividad. Por ende, se considera que la especialización tiene un impacto en las curvas de aprendizaje (Schilling et al., 2003). Al respecto, autores como Glock \& Jaber (2014) asumen una curva de potencia similar a la desarrollada por Wright (1936). Asimismo, se han desarrollado aplicaciones para calcular las CAI en agentes de centros de llamadas tanto para valorar el tiempo de duración de las llamadas entrantes en solucionar solicitudes (Gans et al., 2010), como para evaluar el tiempo en dar solución a requerimeintos técnicos con un conocimiento especializado (; Kim, Krishnan, Argote, Kim, \& Argote, 2016). Por ende, para efectos de este estudio se definirán la CAI en los agentes del centro de llamadas salientes con relación al tiempo definido y el número de ventas realizadas efectivamente.

\section{Metodología}

Este estudio se centra en un centro de llamadas salientes -outband-, es decir, donde los agentes realizan llamadas para contactar a los clientes, con el propósito de ofrecerles un servicio. En este caso, se selecciona una de las campañas que lleva a cabo La Empresa Gestora, que consiste en una tercerización de una empresa de telefonía móvil, donde las llamadas realizadas por los agentes están orientadas a ofrecer un portafolio de servicios de telefonía móvil con diversas opciones, según las ofertas desarrolladas por la empresa. Al momento de realización de esta investigación, esta campaña estuvo integrada por 120 agentes activos. De ella se seleccionó, a través de un muestreo probabilístico, una muestra de 74 agentes -51 agentes antiguos y 23 nuevos agentes-, asumiendo un nivel de confianza del $95 \%$ y un error muestral del 5,8\%. Estos agentes, a su vez, estuvieron agrupados en 3 grupos, cada uno con su supervisor, estando conformados los grupos 1 y 2 por agentes antiguos de la empresa, y el grupo 3 por nuevos agentes.

Para llevar a cabo el análisis propuesto en este artículo, se acudió a la información suministrada por La Empresa. Para la estimación de las CAI se utilizaron datos diarios para el mes de agosto del 2015, ya que durante este mes entraron nuevos agentes a laborar en la empresa en dos momentos diferentes del mes, lo que permitió el cálculo de las CAI tanto para trabajadores con mayor tiempo laborando en La Empresa Gestora, como para los nuevos trabajadores -en el tiempo transcurrido desde su ingreso hasta finalizar el mes-.

Para efectos de esta investigación, las variables utilizadas son variables proxy del modelo seguido. Por consiguiente, para lograr el objetivo de esta investigación, se construyen las siguientes variables con base a lo expuesto por Glock \& Jaber (2014):

$\mathrm{Y}_{\hat{i}}(\mathrm{~T})$ : La medida de rendimiento es el tiempo $\mathrm{T}$ en función del porcentaje de efectividad de ventas realizadas con relación a las llamadas contactadas por el individuo i del grupo j en el día, entendiendo, que es el tiempo transcurrido desde que el individuo i inicia su día laboral y realiza la primera llamada para contactar a un cliente. Se toma la variable tiempo como medida de productividad, debido a que la evaluación de los clientes de un servicio está fuertemente influenciada por el tiempo utilizado para prestar el servicio requerido (Zeithaml, Parasuraman, \& Berry, 1990).

$\mathrm{y} 1, \mathrm{i}:$ Tasa de producción inicial del servicio de venta del individuo en el inicio del período planeado, medido en relación al porcentaje de efectividad y el porcentaje de utilización de los recursos.

$T$ : Tiempo usado en minutos durante las horas laboradas.

$l i:$ : Tasa de aprendizaje del individuo $i, 0<l_{i}<1$

Para llevar a cabo la estimación de las CAI se emplea la función de potencia estándar de curva de aprendizaje. La función estándar es formulada como , tal como se expuso con ante- 
rioridad. Por lo tanto, se lleva a cabo una transformación logarítmica de las variables definidas en este estudio para estimar la función de potencia, con el fin de obtener el siguiente modelo de regresión:

$$
\operatorname{Ln}\left(Y_{1}(T)\right)=\beta 0+\beta 1 \operatorname{Ln}(y 1, i)
$$

\section{Resultados}

Tal como se señaló en el apartado anterior, los agentes estuvieron organizados en 3 grupos de trabajo, cada uno de ellos coordinados por un supervisor, de allí que los resultados se muestran por cada grupo existente. Sin embargo, se aclara que los resultados se organizan de esta manera solo con el fin de diferenciar los grupos a los que pertenece el agente, y no porque se estimen curvas de Aprendizaje Grupal.

Los resultados obtenidos de las CAI calculadas para los agentes de cada grupo muestran que, en la medida que aumenta la experiencia en venta de los agentes, disminuye el tiempo de la llamada contactada, es decir, que la llamada fue atendida por el cliente y el agente pudo ofrecer los servicios de interés de La Empresa y efectuar una venta de esos servicios ofrecidos. Sin embargo, el individuo 1 del grupo 3 (Tabla 3), el signo del es positivo, indicando que al aumentar la experiencia en venta, aumenta el tiempo utilizado por el agente. Además, se observa que los valores de en los modelos de los individuos del grupo 3, son superiores a los obtenidos en los grupos 1 y 2 que hacen referencia a los agentes antiguos.

Por su parte, para algunos resultados de los modelos estimados el Coeficiente de Determinación en la mayoría de los individuos es superior a 0,8 , indicando que la variable es explicada por la variable, que fueron definidas para la estimación del modelo. Sin embargo, los modelos de los agentes 6 y 7 en el grupo 1, los modelos de los agentes 2, 6, 2 y 3, en el grupo 2 son superiores a 0,6 lo que indica una disminución en el grado de asociación entre las variables.

Por otro lado, con respecto al Análisis de Varianza (ANOVA) utilizado para contrastar si existe o no relación significativa entre las variables; se observa que con un nivel de significancia del 0,05 se rechaza la hipótesis nula del estadístico F; lo cual implica que existe una relación lineal entre las variables.

\section{Consideraciones finales}

Este artículo centró su atención en el cálculo de las calcular las curvas de aprendizaje individual de los agentes de un centro de llamadas, con el fin de determinar la relación entre el tiempo utilizado para contactar con llamadas con respecto a las ventas realizadas. Para ello se analizó la información de una muestra de 74 agentes, siguiendo un muestro probabilístico, con un nivel de confianza del $95 \%$ y un error muestral del 5,8\%. Los resultados arrojan que, en la medida que los agentes aumentan su experiencia en ventas, disminuye el tiempo utilizado para contactar a los clientes. Asimismo, se observa que en los individuos del grupo 3, integrado por trabajadores nuevos en la empresa, los valores de los $\beta_{-} 1$ llegan a ser superiores con relación a los otros individuos. El resultado anterior, sin pretender ser determinante, puede obedecer al aprendizaje que adquieren durante la preparación dada por la empresa, a través de una formación de dos meses con simulaciones relacionadas a la actividad que van a desempeñar.

Para próximas investigaciones realizadas en este tipo de Call Center se deben evaluar otras variables como ausentismo -dado que, para es centro de llamadas, los agentes llegan a presentar tasas de ausentismo significativas-, así como las tasas de retiro. En el caso del ausentismo, éste puede considerarse un factor relevante en las curvas de aprendizaje, puesto que puede ser visto como una consecuencia de que los colaboradores de una empresa no se involucren en el aprendizaje individual, en grupo u organizacional. Por último, de proponen realizar estudios para determinar si las pruebas de selección de personal deberían estar en función de las curvas de aprendizaje de la organización, para determinar si éstas influyen en el retiro de los agentes. 
Aproximación a las Curvas de Aprendizaje Individual: Call Center Colombia

\section{Referencias}

Aguirre, A. (1985). Las curvas de aprendizaje y sus aplicaciones. Cuadernos de Ciencias Económicas Y Empresariales, 16, 121-134.

Aksin, Z., Armony, M., \& Mehrotra, V. (2007). The Modern Call Center : A Multi-. Production and Operations Management Society, 16(6), 665-688. http://doi.org/10.3401/poms.

Almeida, J. (2015). Elastix Call Center para supervisores y Administradores.

Argote, L. (2000). Organizational Learning: Creating, Retaining, and Transferring Knowledge. Administrative Science Quarterly, 45, 622. http://doi.org/10.2307/2667112

Badiru, A. B. (1992). computanional survey of univariate and multivariate learning curve models. IEEE Transactions on Engineering Management, 39(2), 176-188.

Cyert, R., \& March, J. (1963). A Behavioral Theory of the Firm (Englewood).

Dar-El, E. M. (2000). Human Learning: from Learning Curves to Learning Organizations. Kluver Academic Publisher. Massachusetts.

Ellis, H. C. (1965). The transfer of learning. Macmillan Company. Oxford, England.

Fioretti, G. (2007). The organizational learning curve. European Journal of Operational Research, $177(3), 1375-1384$.

Gans, N., Liu, N., \& Mandelbaum, A. (2010). Service times in call centers : Agent heterogeneity and learning with some. Institute of Mathematical Statistics., 6, 99-123. http://doi.org/10.1214/10-IMSCOLL608

Glover, J. H. (1966). Manufacturing progress functions: an alternative model and its comparison with existing functions. International Journal of Production Research, 4, 279.

H. Glock, C., \& Y. Jaber, M. (2014). A group learning curve model with and without worker turnover. Journal of Modelling in Management, 9(2), 179-199. http://doi.org/10.1108/JM2-05-2013-0018

Huber, G. P. (1991). Organizational Learning: The Contributing Processes and the Literatures. Organization Science, 2(1), 88-115. http://doi.org/10.1287/orsc.2.1.88

Kim, Y., Krishnan, R., Argote, L., Kim, Y., \& Argote, L. (2016). The Learning Curve of IT Knowledge Workers in a Computing Call Center. Information Systems Research, 23(23(3-part-2)), 887-902.

Levitt, B; March, J. (1988). Organizational Learning. Annual Review of Sociology, 14(May), 319340. http://doi.org/Doi 10.1146/Annurev.So.14.080188.001535

Lloyd, N. (1971). Productivity Gains with the learning curve. Industrial Managment, 9-12.

Nadier, G., \& Smith, W. (1964). Courbes d'accountumance et processus de fabrication (L'Etude du).

Schilling, M. a., Vidal, P., Ployhart, R. E., \& Marangoni, A. (2003). Learning by Doing Something Else: Variation, Relatedness, and the Learning Curve. Management Science, 49(1), 39-56.

Teplitz, C. J. (1991). NoThe learning curve deskbook: A reference guide to theory, calculations, and applications (Quorum Boo).

Towill, D. R., \& Bevis, F. W. (1972). Manegeral control system based on Learning Curve models. International Journal of Production Research, 11(3), 219-238. 
Wright, T. P. (1936). Factors Effecting the Cost of Airplanes. Journal of Aereonautical Sciences, 3(4), $122-128$.

Zeithaml, V. A., Parasuraman, A., \& Berry, L. L. (1990). Delivering Quality Service: Balancing Customer Expectations and Perceptions. (F. Press, Ed.). New York. 